\title{
Video Article \\ Single Liposome Measurements for the Study of Proton-Pumping Membrane Enzymes Using Electrochemistry and Fluorescent Microscopy
}

\author{
levgen Mazurenko ${ }^{1}$, Nikos S. Hatzakis ${ }^{2}$, Lars J.C. Jeuken ${ }^{1}$ \\ ${ }^{1}$ School of Biomedical Sciences \& the Astbury Centre for Structural Molecular Biology, University of Leeds \\ ${ }^{2}$ Department of Chemistry and Nano-Science Center, University of Copenhagen
}

Correspondence to: Lars J.C. Jeuken at I.j.c.jeuken@leeds.ac.uk

URL: https://www.jove.com/video/58896

DOI: doi:10.3791/58896

Keywords: Biochemistry, Issue 144, cytochrome $b_{3}$, single enzyme, proteoliposomes, pH-sensitive dye, proton translocation, bioelectrochemistry

Date Published: 2/21/2019

Citation: Mazurenko, I., Hatzakis, N.S., Jeuken, L.J. Single Liposome Measurements for the Study of Proton-Pumping Membrane Enzymes Using Electrochemistry and Fluorescent Microscopy. J. Vis. Exp. (144), e58896, doi:10.3791/58896 (2019).

\section{Abstract}

Proton-pumping enzymes of electron transfer chains couple redox reactions to proton translocation across the membrane, creating a protonmotive force used for ATP production. The amphiphilic nature of membrane proteins requires particular attention to their handling, and reconstitution into the natural lipid environment is indispensable when studying membrane transport processes like proton translocation. Here, we detail a method that has been used for the investigation of the proton-pumping mechanism of membrane redox enzymes, taking cytochrome $\mathrm{bo}_{3}$ from Escherichia coli as an example. A combination of electrochemistry and fluorescence microscopy is used to control the redox state of the quinone pool and monitor $\mathrm{pH}$ changes in the lumen. Due to the spatial resolution of fluorescent microscopy, hundreds of liposomes can be measured simultaneously while the enzyme content can be scaled down to a single enzyme or transporter per liposome. The respective single enzyme analysis can reveal patterns in the enzyme functional dynamics that might be otherwise hidden by the behavior of the whole population. We include a description of a script for automated image analysis.

\section{Video Link}

The video component of this article can be found at https://www.jove.com/video/58896/

\section{Introduction}

Information about enzyme mechanisms and kinetics is usually obtained on the ensemble or macroscale level with enzyme population in the thousands to millions of molecules, where measurements represent a statistical average. It is known, however, that complex macromolecules such as enzymes may demonstrate heterogeneity in their behavior and molecular mechanisms observed at the ensemble level are not necessarily valid for every molecule. Such deviations on the individual molecule scale have been extensively confirmed by studies of single enzymes with a variety of methods emerging during the last two decades ${ }^{1}$. Notably, fluorescence detection of individual enzyme activity has been used to investigate heterogeneity of enzymes activity ${ }^{2,3}$ or discover the so-called memory effect (periods of high enzymes activity succeeded by periods of low activity and vice versa) ${ }^{4,5}$.

Many single enzyme studies require that the enzymes are immobilized on the surface or spatially fixed in another way to remain sufficiently long in the field of view for continuous observation. Enzyme encapsulation into liposomes has been shown to enable enzyme immobilization while preventing any negative impact due the surface-enzyme or protein-protein interactions ${ }^{6,7}$. In addition, liposomes offer a unique possibility to study single membrane proteins in their natural lipid bilayer environment ${ }^{8,9,10}$

A class of membrane proteins, transporters, exercises a directional translocation of substances across the cell membrane, a behavior that can only be studied when proteins are reconstituted into the lipid bilayers (e.g., liposomes) ${ }^{11,12,13}$. For example, proton translocation, exhibited by several enzymes of prokaryotic and eukaryotic electron transport chains, plays an important role in cellular respiration by creating a proton-motive force used for ATP synthesis. In this case, the proton pumping activity is coupled to the electron transfer, although the detailed mechanism of this process often remains elusive.

Recently, we demonstrated the possibility to couple fluorescent detection with electrochemistry to study proton pumping activity of single enzymes of the terminal ubiquinol oxidase of Escherichia coli (cytochrome $b_{3}$ ) reconstituted in the liposomes ${ }^{14}$. This was achieved by encapsulation of a pH-sensitive membrane-impermeable fluorescent dye into the lumen of liposomes prepared from E. coli polar lipids (Figure 1A). The protein amount was optimized so that most liposomes either contained no or only one reconstituted enzyme molecule (according to Poisson distribution). The two substrates of cytochrome $b_{3}$ were provided by adding ubiquinone to the lipid mix that formed the liposomes and (ambient) oxygen in solution. The liposomes are then sparsely adsorbed on a semi-transparent ultra-smooth gold electrode, covered with a selfassembled monolayer of 6-mercaptohexanol. Finally, the electrode is mounted on the bottom of a simple spectroelectrochemical cell (Figure 1B). Electrochemical control of the quinone pool redox state allows one to flexibly trigger or to stop the enzymatic reaction at any moment, while the $\mathrm{pH}$-sensitive dye is used to monitor $\mathrm{pH}$ changes inside the lumen of the liposomes as a result of proton translocation by the enzymes. By using the fluorescence intensity of a second, lipid-bound fluorescent dye, the size and volume of individual liposomes can be determined and 
thus the quantification of enzyme proton pumping activity. Using this technique, we notably found that cytochrome $\mathrm{bo}_{3}$ molecules are able to enter into a spontaneous leak state that rapidly dissipates the proton motive force. The goal of this article is to introduce the technique of single liposome measurements in detail.

\section{Protocol}

\section{Preparation of a Lipid/UQ-10/FDLL Mix}

NOTE: E. coli lipids used for the liposomes' preparation should be aliquoted and thoroughly mixed with ubiquinone-10 (enzyme substrate) and long-wavelength fluorescent dye-labeled lipids (for liposomes size determination) prior to the reconstitution.

1. Using a glass syringe, transfer $200 \mu \mathrm{L}$ of chloroform stock of lipid polar extract from Escherichia coli $(25 \mathrm{mg} / \mathrm{mL})$ into glass vials to $\mathrm{make} 5 \mathrm{mg}$ aliquots.

2. Add $50 \mu \mathrm{L}$ of $1 \mathrm{mg} / \mathrm{mL}$ ubiquinone-10 (UQ-10; in chloroform) to the lipids to make the final ratio of UQ-10: lipids $1: 100$ (1\% w/w)

3. Add $20 \mu \mathrm{L}$ of $1 \mathrm{mg} / \mathrm{mL}(0.4 \% \mathrm{w} / \mathrm{w})$ of a long-wavelength fluorescent dye-labeled lipid (FDLL) to the lipids/UQ-10 mix.

4. Homogenize the chloroform solution by short vortexing and evaporate most of the chloroform under a gentle nitrogen or argon flow. Remove the chloroform traces entirely by further evaporation under vacuum for at least $1 \mathrm{~h}$.

NOTE: Lipid aliquots can be stored under an inert atmosphere at $-20^{\circ} \mathrm{C}$ for several months.

\section{Reconstitution of Cytochrome $\mathrm{bo}_{3}$}

NOTE: For the purification of cytochrome $b_{3}$ from $E$. coli, follow the protocol from Rumbley et al. ${ }^{15}$ To ensure high purity of natively-folded enzyme samples, add size-exclusion chromatography after the affinity purification step described by Rumbley et al. ${ }^{15}$

1. Add $312.5 \mu \mathrm{L}$ of $40 \mathrm{mM}$ MOPS- $\mathrm{KOH} / 60 \mathrm{mM} \mathrm{K}_{2} \mathrm{SO}_{4}, \mathrm{pH} 7.4$, to one aliquot of lipids/UQ-10/FDLL dry mix (5 mg, step 1.4) and mix with vortex till the lipid film is fully resuspended, followed by 2 min of treatment in an ultrasonic bath.

2. Add $125 \mu \mathrm{L}$ of $25 \mathrm{mM}$ 8-hydroxypyrene-1,3,6-trisulphonic acid (HPTS), a pH-sensitive fluorescent dye that needs to be encapsulated inside the liposomes.

3. Add $137.5 \mu \mathrm{L}$ of $250 \mathrm{mM} \mathrm{n}$-octyl $\beta$-D-glucopyranoside (OGP) surfactant, mix using vortex and sonicate in an ultrasonic water bath for $10 \mathrm{~min}$ to ensure all lipids are solubilized into surfactant micelles. Transfer the dispersion into a $1.5 \mathrm{~mL}$ plastic tube. NOTE: Cloudy suspension of lipids should become transparent after the solubilization with surfactant.

4. Add the required amount of cytochrome $\mathrm{bo}_{3}$ (see the note below) and add ultrapure water to make a total volume of $50 \mu \mathrm{L}$ (cytochrome $\mathrm{bo}_{3}$ solution plus water). Incubate at $4{ }^{\circ} \mathrm{C}$ for 10 min on a roller mixer.

NOTE: Typical amount for single enzyme conditions is $0.1-0.2 \%$ (w/w protein-to-lipid, i.e. $5-10 \mu g$ of protein), although it can be increased till $1-2 \%(50-100 \mu \mathrm{g}$ of protein) if the goal is to observe only the electrochemical activity (see below). As a negative control, liposomes without cytochrome $\mathrm{bo}_{3}$ can be prepared.

5. Weigh $2 \times 50 \mathrm{mg}$ and $2 \times 100 \mathrm{mg}$ of polystyrene microbeads into four $1.5 \mathrm{~mL}$-tube caps and close with paraffin film to prevent drying. NOTE: Before use, polystyrene microbeads should be washed with methanol, water and stored in water according to the manufacturer's manual.

NOTE: If a water/microbead mixture is used, polystyrene microbeads can conveniently be transferred to the cap with a micropipette with a wide tip. Water can then be removed from the microbeads using a micropipette with a thin tip.

6. Add the $1^{\text {st }} \mathbf{5 0} \mathbf{~ m g}$ of polystyrene microbeads into the reconstitution mixture (step 2.4) by putting the cap with polystyrene microbeads on the $1.5 \mathrm{~mL}$-plastic tube with dispersion and performing a short spin for a couple of seconds. Incubate at $4{ }^{\circ} \mathrm{C}$ on a roller mixer for polystyrene microbeads to adsorb the surfactant for $\mathbf{3 0} \mathrm{min}$.

1. Repeat the additions of polystyrene microbeads and incubations as follows: add $\mathbf{5 0} \mathbf{~ m g}$ of microbeads for $\mathbf{6 0}$ min of incubation; add $100 \mathrm{mg}$ of microbeads for $\mathbf{6 0} \mathrm{min}$ of incubation; and add $\mathbf{1 0 0} \mathrm{mg}$ of microbeads for $\mathbf{1 2 0}$ min of incubation.

NOTE: The solution above the settled microbeads will turn translucent during step 2.6 as proteoliposomes are formed.

7. Separate the proteoliposome solution from the polystyrene microbeads using a micropipette with a thin tip. Dilute the dispersion in $90 \mathrm{~mL}$ of $20 \mathrm{mM}$ MOPS- $\mathrm{KOH} / 30 \mathrm{mM} \mathrm{K}_{2} \mathrm{SO}_{4}, \mathrm{pH} 7.4$ (MOPS buffer) and transfer in a Ti45 ultracentrifuge tube.

8. Ultracentrifuge the dispersion using Type $45 \mathrm{Ti}$ rotor at $125,000 \times \mathrm{g}$ (at $\left.\mathrm{r}_{\max }\right)$ for $1 \mathrm{~h}$ to pellet the proteoliposomes. NOTE: Smaller centrifuge tubes can be used although the dilution in large buffer volume helps to reduce the concentration of the nonencapsulated HPTS in the final suspension.

9. Discard the supernatant, rinse the pellets with $20 \mathrm{mM} \mathrm{MOPS}-\mathrm{KOH} / 30 \mathrm{mM} \mathrm{K}_{2} \mathrm{SO}_{4}, \mathrm{pH} 7.4$ buffer (without resuspending the pellet). After discarding the rinsing buffer, re-suspend the proteoliposomes in $500 \mu \mathrm{L}$ of MOPS buffer by pipetting it back and forth with thin tip micropipette. Then transfer to a $1.5 \mathrm{~mL}$ plastic tube.

10. Centrifuge the suspension for $5 \mathrm{~min}$ at $12,000 \mathrm{xg}$ to remove the debris. Transfer the supernatant (reconstituted proteoliposomes) into a new vial.

11. Store the reconstituted proteoliposomes dispersion at $4{ }^{\circ} \mathrm{C}$ overnight and use within 2 days.

\section{Fabrication of Semi-Transparent Gold Electrodes}

NOTE: The smooth gold surface is obtained by a template stripping method of a $30 \mathrm{~nm}$-thick layer of $99.99 \%$ gold from an atomically smooth silicon wafer. The small thickness of the gold layer is important since it must be semi-transparent to permit fluorescence observation. Details of gold evaporation (physical vapor deposition, PVD) can be find elsewhere ${ }^{16}$ and only template-stripping is covered here. Alternatively, ultrasmooth gold chips can be purchased elsewhere (see Table of Materials). 
1. Glue up to 9 glass cover slips $(0.17 \mathrm{~mm}$ thick) onto the evaporated gold surface using bi-component low-fluorescence epoxy. Cure the glue at $80^{\circ} \mathrm{C}$ for $4 \mathrm{~h}$.

2. Just before modification with a self-assembled monolayer (step 4), detach the glass cover slips from the silicon wafers with a blade. Due to the thinness of the cover slips, take care when detaching the cover slips to not crack or break the glass slides.

\section{Modification of the Gold Surface with Self-Assembled Monolayer (SAM)}

1. Prepare $5 \mathrm{~mL}$ of water solution of $1 \mathrm{mM} 6$-mercaptohexanol $(6 \mathrm{MH})$.

2. Dip freshly detached gold-coated cover slips (step 3.1) into $6 \mathrm{MH}$ solution and leave at $20-25^{\circ} \mathrm{C}$ overnight (>16 h) to form the SAM. NOTE: Thiol solutions have an unpleasant smell, so a closed vessel should be used when incubating the gold-coated cover slip.

3. The next day, remove the gold-coated cover slip from the $6 \mathrm{MH}$ solution, wash briefly with water or methanol and then with isopropanol. Dry under a gentle gas flow.

\section{Electrochemical Testing of Proteoliposomes Activity}

NOTE: The electrochemical activity of enzyme is first verified on a closely packed liposomes layer (step 5) and lower vesicle coverages are used in single vesicles experiment to measure $\mathrm{pH}$ changes in the lumen of the liposomes (step 6).

1. Assemble the gold-coated cover slip in a spectro-electrochemical cell (see Figure 1). Make contact to the gold with a flat wire outside an area defined by a rubber O-ring.

2. Add $2 \mathrm{~mL}$ of the electrolyte buffer solution and place the reference and auxiliary electrodes in the cell.

NOTE: As further discussed in the Discussion section, references electrodes without chloride are preferred to prevent formation of $\mathrm{Au}(\mathrm{I}) \mathrm{Cl}$ during electrochemistry. Here, a $\mathrm{Hg} / \mathrm{Hg}_{2} \mathrm{SO}_{4}\left(\right.$ sat. $\mathrm{K}_{2} \mathrm{SO}_{4}$ ) reference electrode is used and potentials are given versus Standard Hydrogen Electrode (SHE) using $0.658 \mathrm{~V}$ vs SHE for the $\mathrm{Hg} / \mathrm{Hg}_{2} \mathrm{SO}_{4}$ (sat. $\mathrm{K}_{2} \mathrm{SO}_{4}$ ).

3. Run electrochemical impedance spectroscopy $(0.1 \mathrm{~Hz}-100 \mathrm{kHz})$ at open cell potential (OCP) potential to assess the quality of SAM. Convert impedance values to admittance and divide by $2 \pi \omega$ to plot a Cole-Cole plot, where $\omega$ is the frequency (see the following references ${ }^{16,17,18}$ for details on converting and interpreting impedance spectra).

NOTE: Compact and dense SAMs of $6 \mathrm{MH}$ should give a close to semi-circle Cole-Cole plot and give capacitance values in the range 2.5-3.0 $\mu \mathrm{F} / \mathrm{cm}^{2}$ (Figure 2A). If significant deviation from semi-circle shape or out-of-range capacitance values are obtained, change the electrode.

4. Run blank cyclic voltammograms (CVs) with scan rates 100 and $10 \mathrm{mV} / \mathrm{s}$ in the potential region $-0.3-0.8 \mathrm{~V}$. A typical CV is shown on (Figure 2B, dashed line).

NOTE: This should demonstrate almost pure capacitive behavior and an absence of significant faradaic current, even under ambient oxygen conditions as used here.

5. Add proteoliposomes $\left(0.5 \mathrm{mg} / \mathrm{mL}\right.$ final lipids concentration, $1-2 \%(\mathrm{w} / \mathrm{w})$ ratio of cytochrome $\mathrm{bo}_{3}$ to lipid) to the electrochemical cell and mix slightly with a pipette. Wait until the adsorption of proteoliposomes on the electrode surface is finished (30-60 min at room temperature). NOTE: Cyclic voltammograms (CVs) at $10 \mathrm{mV} / \mathrm{s}$ can be run during the proteoliposomes adsorption to follow the process. The adsorption is finished when consecutive CVs stop changing. Information about CV techniques can be found in the following textbooks. ${ }^{19,20}$

6. Wash the cell by changing the buffer solution at least 10 times but avoid leaving the electrode surface completely dry.

7. Run the electrochemical impedance spectroscopy at OCP (Figure 2A, blue line) to confirm the SAM on the gold electrode remains unchanged and $\mathrm{CVs}$ with scan rates 10 and $100 \mathrm{mV} / \mathrm{s}$ to observe catalytic ubiquinol oxidation (and oxygen reduction) by cytochrome $\mathrm{bo}_{3}$ at onset potentials of electrochemical quinone reduction about $0 \mathrm{~V}$ vs SHE) (Figure 2B).

\section{Detection of Enzymatic Proton Pumping by Fluorescence Microscopy}

1. Modify the gold electrode as in step 5 but using $100 x$ less proteoliposomes compared to step 5.5 (i.e., $5 \mu \mathrm{g} / \mathrm{mL}$ ). For single enzyme studies, reduce the cytochrome $b_{3}$ to lipid ratio to $0.1-0.2 \%(\mathrm{w} / \mathrm{w})$.

NOTE: Liposomes will sparsely adsorb on the electrode surface enabling single vesicle monitoring by fluorescence microscopy. Under these single-enzyme conditions, the amount of enzyme immobilized on the electrode surface is insufficient for the observation of a catalytic current.

2. Place the electrochemical cell on the oil objective $(60 \mathrm{X})$ of an inverted fluorescence microscope with a drop of immersion oil. Using appropriate filters for FDLL fluorescence, focus on the electrode surface. Single liposomes should appear as bright spots at the diffraction limit of the microscope/objective. Take an image of FDLL fluorescence.

3. Switch to the one of HPTS fluorescence filter sets on the microscope to verify that HPTS fluorescence is clearly visible and distinguishable from the background (at the chosen exposure time, see step 6.4). Increase the light intensity if it is not the case.

4. Program the microscope software to perform a timed image acquisition by alternating two HPTS filter sets (menu Applications | Define/Run ND Acquisition). Set the delay between image acquisitions at minimum. In this experiment, use a $1 \mathrm{~s}$ exposure and $0.3 \mathrm{~s}$ delay (due to the turret movement).

NOTE: The ratio between fluorescence intensities at these two channels will be later converted to $\mathrm{pH}$ inside the liposomes at each time point. The duration of the acquisition can vary according to the expected $\mathrm{pH}$ change rate; $5 \mathrm{~min}$ is used in this article.

5. Adjust the settings of the potentiostat to change the potential during the image acquisition. For example, in this experiment, use the following sequence: 0 - 60 s: no potential applied (i.e. OCP); $60-180 \mathrm{~s}$ : $-0.2 \mathrm{~V}$ (vs SHE); $180-300 \mathrm{~s}$ : $0.4 \mathrm{~V}$ (vs SHE). In this case, only the applied potential in the second phase $(-0.2 \mathrm{~V}$ vs $\mathrm{SHE})$ is sufficient to efficiently reduce the quinone pool.

6. Run simultaneously the timed images acquisition (microscope) and the potential sequence (potentiostat) by manually starting both measurements at the same time.

NOTE: The experiment can be repeated on the same electrode several time by moving the microscope stage to a different area on the surface. The delay between the acquisitions should be at least 5-10 min to insure complete pH equilibration of liposomes on the surface. Different durations and potential patterns can be applied depending on the need, although imaging time is limited due to photobleaching of HPTS during acquisition. 


\section{Analysis of Fluorescence Images}

NOTE: A typical experiment produces a set of images with a time step (e.g., $2.6 \mathrm{~s}$ ) for each of the two channels, i.e., (duration * $2 / 2.6$ ) images. An example of such image set recorded during a single enzyme experiment can be accessed via the Research Data Leeds repository ${ }^{21}$. An image treatment consists of several steps by using Fiji (Image J) and high-level mathematical analysis programming language software (henceforth referred to as scripting software, see Table of Materials for details).

1. Use Fiji to separate time lapse file, align images and save as separate channels and timeframes.

1. Open time lapse file using the Bioformats importer provided within Fiji (macro command: run ("Bio-Formats Importer")).

2. Use the plugin StackReg to align each frame to the first one to account for possible stage movements or thermal drift occurred during the acquisition (macro command: run("StackReg ", "transformation=Translation")).

3. Save separate uncompressed image files for each channel and each time step in TIFF format into a single folder.

4. Extract the exact time stamps for each frame from the time lapse file metadata and save them as a CSV-file in the same folder as the images. Alternatively, extract time stamps using the acquisition software and save manually into a CSV-file using a spreadsheet software.

NOTE: The folder with images and time stamps is now ready to be analyzed by the scripting software. Steps 7.1.1. - 7.1.4. can be automatized using a Fiji script (a script written in Python for batch processing of time lapse files is provided, use "Ctrl-Shift-N" (in Windows), then File | Open to load the script).

2. Use scripting software for automatized processing of the images. Load the provided code to the software and click Run to end. When prompted, select the folder containing the images from step 7.1.

NOTE: A script for analysis is provided as mlx-format live script, with extensive comments, to identify single liposomes, fit them to 2DGaussian function, filter them and quantify the $\mathrm{pH}$ values at each point of time. The following sub-steps are executed automatically by the script.

1. Load all time frames images for a single experiment into the memory.

2. Average all images for a single channel (select the channel with the highest fluorescence intensity) and use the averaged image to identify all maximums that might correspond to the single liposomes.

3. Fit the identified maximums on the averaged image to the 2D-Gaussian function and save the resulted fit parameters for each liposome (see Figure 4A for fitted liposome example).

4. Filter the maximums according to the expected single liposomes criteria, such as size, circularity and intensity. Reject liposomes that are poorly fitted due to low signal to noise, closely neighboring liposome or being too close to the image edge.

5. Load time stamps from the external file and fit each filtered liposome to the 2D-gaussian function on each time frame image separately. NOTE: The use of parallel programming and multicore CPU can significantly enhance the calculation speed at this stage.

6. Filter the liposomes again according to the similar criteria as in step 7.2.4 but applied to each time frame.

7. On each time step, define the fluorescence intensity ratio of a liposome as the ratio of volumes enclosed by fitted 2D-Gaussian function at two channels. Calculate the $\mathrm{pH}$ values from the ratio of intensities determined from the two HPTS channels and using a calibration curve (step 8). Plot resulting $\mathrm{pH}$-time curves for the liposomes and observe their $\mathrm{pH}$ change when the potential is applied.

\section{Performing a Calibration Curve of HPTS Fluorescence}

NOTE: To convert HPTS fluorescence ratio to an intravesicular $\mathrm{pH}$, a calibration curve must be first established that would take into account particular conditions of experiments such as gold transmittance, filters quality, etc. This step has to be performed only once or twice and the calibration data can be used as long as the setup and measurement parameters remain the same in step 6.

1. Prepare an electrode with sparsely adsorbed liposomes (without cytochrome $b_{3}$ as described in Steps 5 and 6.1).

2. Add $2 \mu \mathrm{L}$ of $0.1 \mathrm{mg} / \mathrm{mL}$ gramicidin solution in ethanol to $2 \mathrm{~mL}$ of buffer to create the concentration $100 \mathrm{ng} / \mathrm{mL}$.

3. Capture two fluorescence images for the two HPTS channels.

4. Change the $\mathrm{pH}$ of the cell by addition of small aliquots of $1 \mathrm{M} \mathrm{HCl}$ or $1 \mathrm{M} \mathrm{H}_{2} \mathrm{SO}_{4}$. Measure the $\mathrm{pH}$ of the buffer with a standard $\mathrm{pH}$ meter and capture two fluorescence images for the two HPTS channels.

5. Repeat steps 8.4 for the $\mathrm{pH}$ range 6 to 9 .

6. Using the algorithm from 7.2, fit, filter and calculate the average HPTS fluorescence ratio of individual liposomes at every pH. Take the HPTS ratio average over all liposomes.

7. Fit the resulting $\mathrm{pH}-$ ratio dependence to the following equation:

$$
p H=p K_{a}+\log \left(\frac{\text { Ratio }-R_{a}}{R_{b}-\text { Ratio }}\right) \text {, where } p K_{a}, R_{a} \text { and } R_{b} \text { are fitting parameters. }
$$

8. Use $p K_{a}, R_{a}$ and $R_{b}$ to convert the HPTS ratio of individual liposomes in step 7.2.7. to $\mathrm{pH}$ values.

\section{Representative Results}

The quality of the gold-modified cover slip (the electrode) with a SAM of $6 \mathrm{MH}$ is checked before each experiment with electrochemical impedance spectroscopy. Figure 2A shows representative Cole-Cole plots measured using electrochemical impedance spectroscopy before and after liposomes are adsorbed. If the quality of SAM is sufficient, impedance spectroscopy should demonstrate an almost pure capacitive behavior resulting in a semi-circle Cole-Cole plot. The diameter of the semi-circle in a Cole-Cole plot equals the double layer capacitance of the electrode surface, which should be in the $2.5-3.0 \mu \mathrm{F} / \mathrm{cm}^{2}$ range. Note that the capacitance should not change significantly upon liposomes adsorption, although a slight increase in capacitance might be observed (high liposomes coverage, Figure $2 \mathbf{A}$, bottom) or a slight shift in impedance can be observed at low frequencies (low liposome coverages, Figure 2A, top). 
Electrochemistry can be used to test the catalytic activity of cytochrome $\mathrm{bo}_{3}$ in the proteoliposomes, where catalytic currents measured with cyclic voltammetry reflect the ubiquinol-oxygen oxidoreductase activity. However, significant catalytic currents can only be detected at high quantities of adsorbed proteoliposomes and a closely packed layer of proteoliposomes on the gold-modified cover slip is needed. Figure 2B demonstrates representative cyclic voltammograms (CVs) of the electrode before and after liposomes adsorption with either low or high liposome coverage. No faradaic current is observed on the blank CV because oxygen reduction by the bare gold electrode is blocked by the SAM. When the surface is saturated with liposomes with high cytochrome $b_{3}$ content $(1.3 \%(\mathrm{w} / \mathrm{w})$ in this case), a clear catalytic wave due to oxygen reduction is observed with an onset potential of $0 \mathrm{~V}$, i.e., the potential of ubiquinone reduction (Figure 2B, blue line). The ubiquinone pool acts both as the natural substrate for the enzyme and as an electron mediator, transferring electrons from the enzyme to the electrode surface. We note that under high liposome coverage, no distinction of individual vesicles is possible by microscopy and lower coverage is needed for single liposome studies. At low liposome coverage (but high protein to lipid ratio), the catalytic current is significantly reduced, barely distinguishable from background (Figure 2B, red line). Note that under single enzyme conditions (low protein to lipid ratio), the catalytic current is even lower and cannot be measured reliably.

Figure 3 shows fluorescence images of liposomes adsorbed on the electrodes at three different coverages. All images were taken in identical light and exposure conditions and their brightness was adjusted equally to enable direct comparison. The dye-containing-liposomes are visible on the images as bright spots. The central part of the image was photobleached for a couple of minutes to reveal background fluorescence level (we note that FDLL is relatively photostable and is not photobleached completely in Figure 3). The images on two HPTS channels are superimposable, where the ratio between the two channels $(410 / 535$ and $470 / 535)$ corresponds to $\mathrm{pH} 7.4$ used in this experiment. A larger number of liposomes are visible with the FDLL channel, which indicates the presence of liposomes that have no HPTS encapsulated. The difference between the HPTS and FDLL channels is more pronounced at higher coverages, possibly because at high liposome coverage, liposomes are more likely to burst or fuse on the surface.

The image analysis requires the alignment of frames (against the first frame) using the ImageJ, plugin StackReg. The alignment is indispensable in most situations since a slight thermal drift of the sample usually occurs within the timescale of the experiment, changing the liposome coordinates. All further analysis is performed using a scripting software code. This code performs automatic liposome identification. As the size of liposomes are below the Abbe diffraction limit, their fluorescence is seen as a point spread function much larger than the actual liposome diameter (Figure 4A). The code fits the fluorescence intensity of each vesicle on two channels to a 2D-Gaussian function (Figure 4A) and calculates their volumetric intensity ratio that can be converted to $\mathrm{pH}$ using a calibration curve. By performing these actions on all time frames, the $\mathrm{pH}$ is obtained inside every vesicle within timescale of the experiment (Movie 1). Figure 4B shows the medians of all vesicle $\mathrm{pH}$ changes within a single image when cytochrome $b_{3}$ content is $1.3 \%$. An increase in $\mathrm{pH}$ (proton pumping) is clearly visible when a potential between -0.1 and $-0.3 \mathrm{~V}$ is applied, but not for $0 \mathrm{~V}$ since the latter is not sufficient to reduce the quinone pool. When cytochrome $b_{3} \mathrm{O}_{3}$ content is much lower (protein-to-lipid ratio of $0.1 \%$, Figure 4C), the median curves become almost indistinguishable from those of empty liposomes (Figure 4D). The difference becomes evident when considering individual $\mathrm{pH}$ traces of random liposomes (Figure 5, 6 and 7). While liposomes without cytochrome $\mathrm{bo}_{3}$ display no significant $\mathrm{pH}$ changes with respect to noise (Figure 7), a selection of liposomes show an increase (dominantly) or a decrease of $\mathrm{pH}$ when a potential is applied that actives cytochrome $\mathrm{bo}_{3}$ (Figures $\mathbf{6}$, grey zone). The obvious difference in $\mathrm{pH}$-traces between liposomes is consistent with the low protein-to-lipid ratio, where cytochrome $b o_{3}$ is only present in a small subset of liposomes activity. The prevalence of a pH increase over decrease is explained by the reconstitution method that favors an "outward" orientation of enzyme molecules (ca. $75 \%$ ). The fraction of liposomes that display a pH change increases when the cytochrome $\mathrm{bo}_{3}$ to lipid ratio is higher (Figure 5). Note also that some liposomes stop proton pumping and enter into proton dissipation mode before the end of the potential application. We attribute this behavior to the cytochrome $b_{3}$ molecules entering a "leak state", which allows protons to flow back into the liposome lumen ${ }^{14}$.

The further analysis of the $\mathrm{pH}$ traces including their fitting and determination of proton pumping/leaking rates can be done using a script we have published previously ${ }^{14,22}$ and can be obtained from the Research Data Leeds Repository ${ }^{23,24}$.
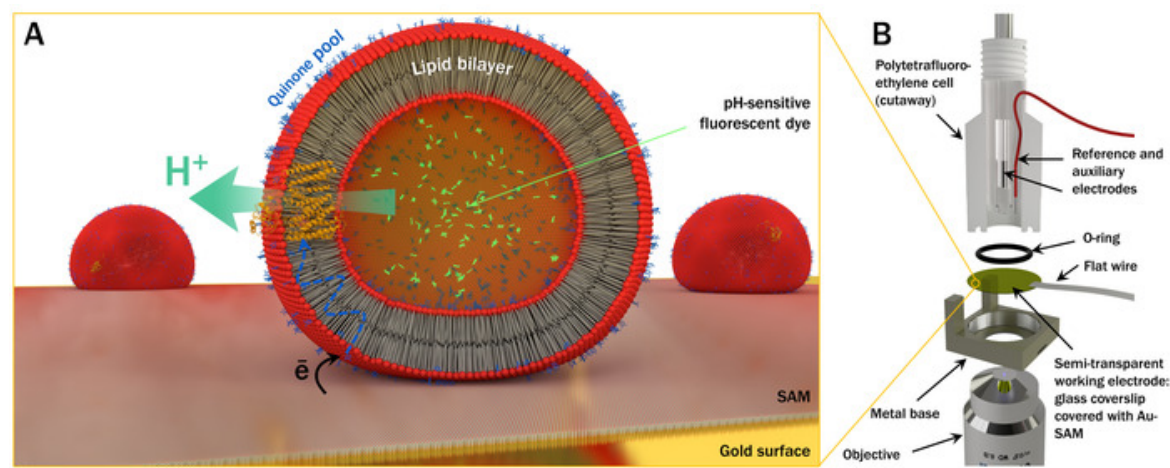

Figure 1: Principle of the method. (A) Principle of single enzyme activity monitoring and (B) the scheme of the experimental setup used in this work with a cutaway to demonstrate an internal part of the cell. Please click here to view a larger version of this figure. 

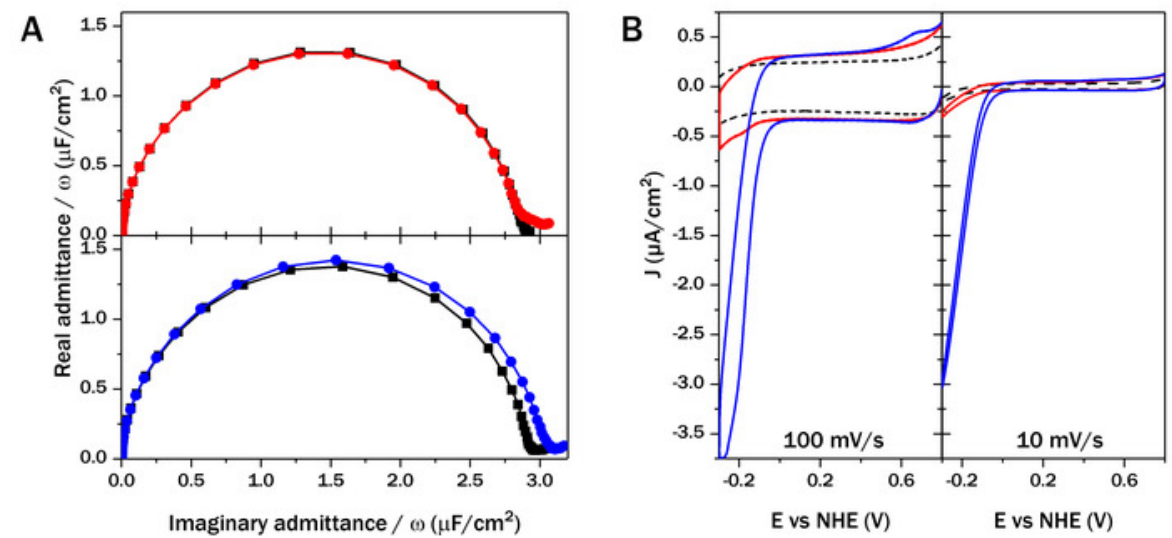

Figure 2: Electrochemical response of liposomes. (A) Cole-Cole plots measured at OCP ( $0.22 \mathrm{~V}$ vs SHE; black squared line) before and after liposomes $\left(1.3 \% \mathrm{w} / \mathrm{w}\right.$ cytochrome $\mathrm{bo}_{3}$ ) adsorption from solutions at concentrations of $5 \mu \mathrm{g} / \mathrm{mL}$ (red circle line) and $500 \mu \mathrm{g} / \mathrm{mL}$ (blue circle line).

(B) Cyclic voltammograms at $100 \mathrm{mV} / \mathrm{s}$ (left panel) and $10 \mathrm{mV} / \mathrm{s}$ (right panel) of Au-SAM (black dashed line) before and after liposomes (1.3\% w/ w cytochrome $\mathrm{bo}_{3}$ ) adsorption from solutions at concentrations of $5 \mu \mathrm{g} / \mathrm{mL}$ (red line) and $500 \mu \mathrm{g} / \mathrm{mL}$ (blue line). Please click here to view a larger version of this figure.

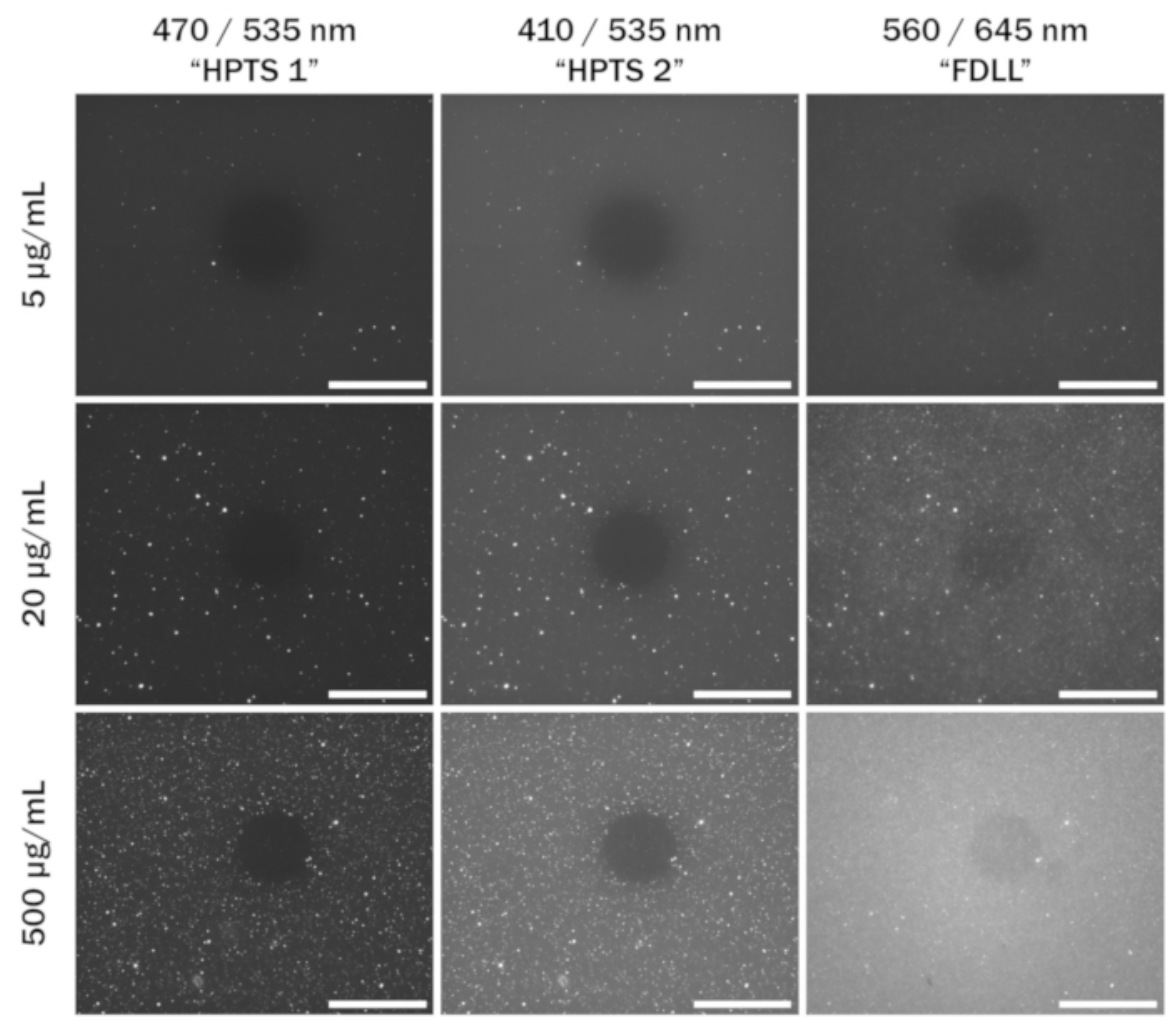

Figure 3: Fluorescence microscopy of liposomes. Fluorescence images of liposomes at different surface coverages: surfaces incubated for $30 \mathrm{~min}$ with a liposomes solution of $5 \mu \mathrm{g} / \mathrm{mL}$ (top row), $20 \mu \mathrm{g} / \mathrm{mL}$ (middle row) and $500 \mu \mathrm{g} / \mathrm{mL}$ (bottom row). Left column corresponds to $470 / 535 \mathrm{~nm}$ excitation/emission filter setup ( $1^{\text {st }}$ HPTS channel); middle column $-410 / 535 \mathrm{~nm}\left(2^{\text {nd }}\right.$ HPTS channel); right column - 560/645 $\mathrm{nm}$ (FDLL channel). The images were taken at magnification $90 \mathrm{X}$ (60X objective and $1.5 \mathrm{X}$ magnification by the microscope prior to the camera), $1 \mathrm{~s}$ exposure and similar light intensity. Scale bars correspond to $50 \mu \mathrm{m}$. Please click here to view a larger version of this figure. 
A
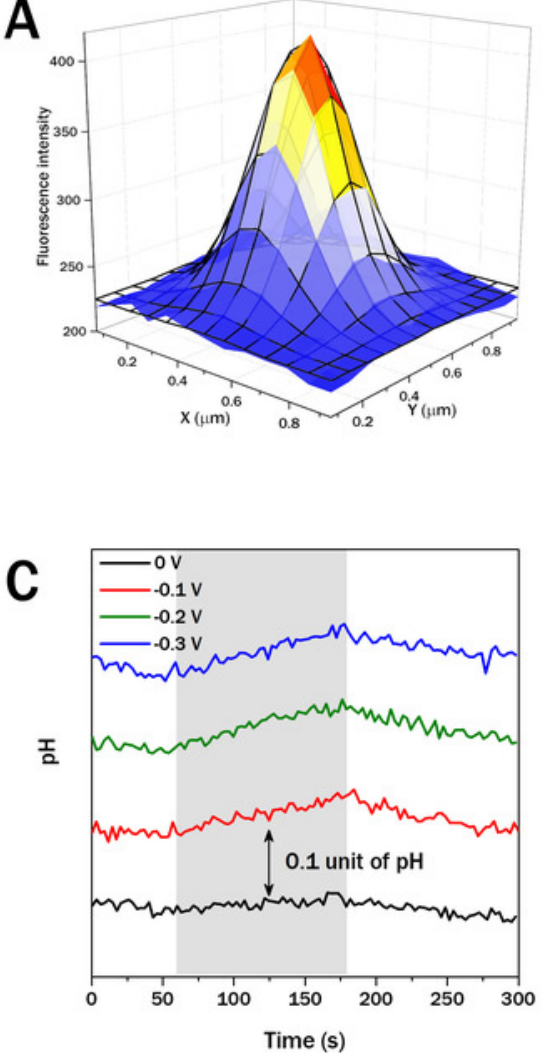
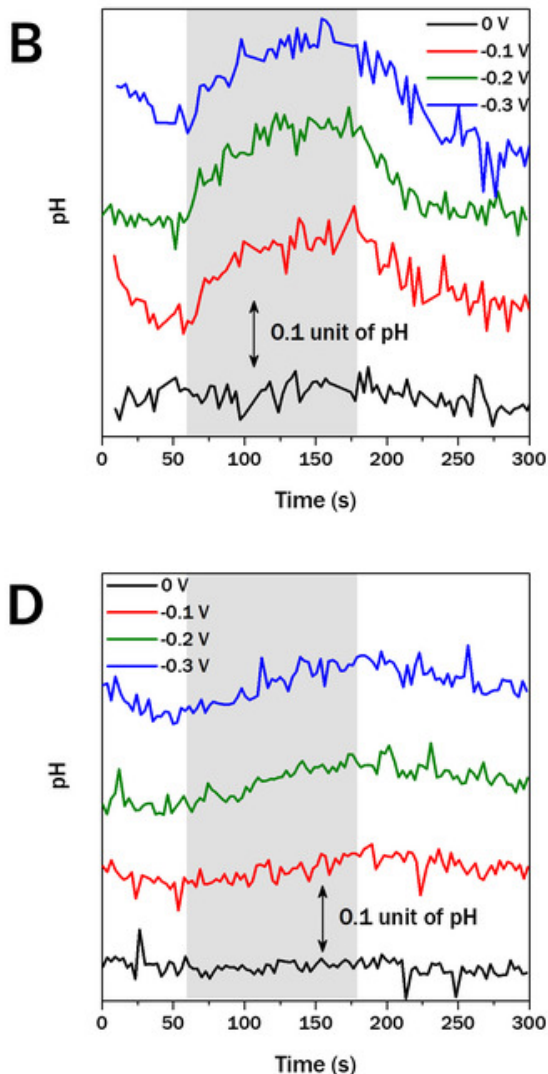

Figure 4: Liposome identification and pH change. (A) 3D-view of a part of fluorescence image containing a typical single liposome. Its fluorescence intensity is seen as a point spread function (color surface) that is fitted to a 2D-Gaussian function (black mesh). (B-D) pH, displayed as the median of all vesicles within single image area (typically several hundred) at different applied potentials. From 0-60 s, no potential is applied (OCP). Between 60 and $180 \mathrm{~s}$ (grey zone) different potentials were applied: $0 \mathrm{~V}$ (black), $-0.1 \mathrm{~V}$ (red), $-0.2 \mathrm{~V}(\mathrm{green}),-0.3 \mathrm{~V}$ (blue). Between 180 and $300 \mathrm{~s}, 0.4 \mathrm{~V}$ vs. SHE was applied. The cytochrome $b o_{3}$ to lipid ratios were (B) $1.3 \%$, (C) $0.1 \%$, (D) $0 \%$. The traces are offset for clarity. Please click here to view a larger version of this figure.

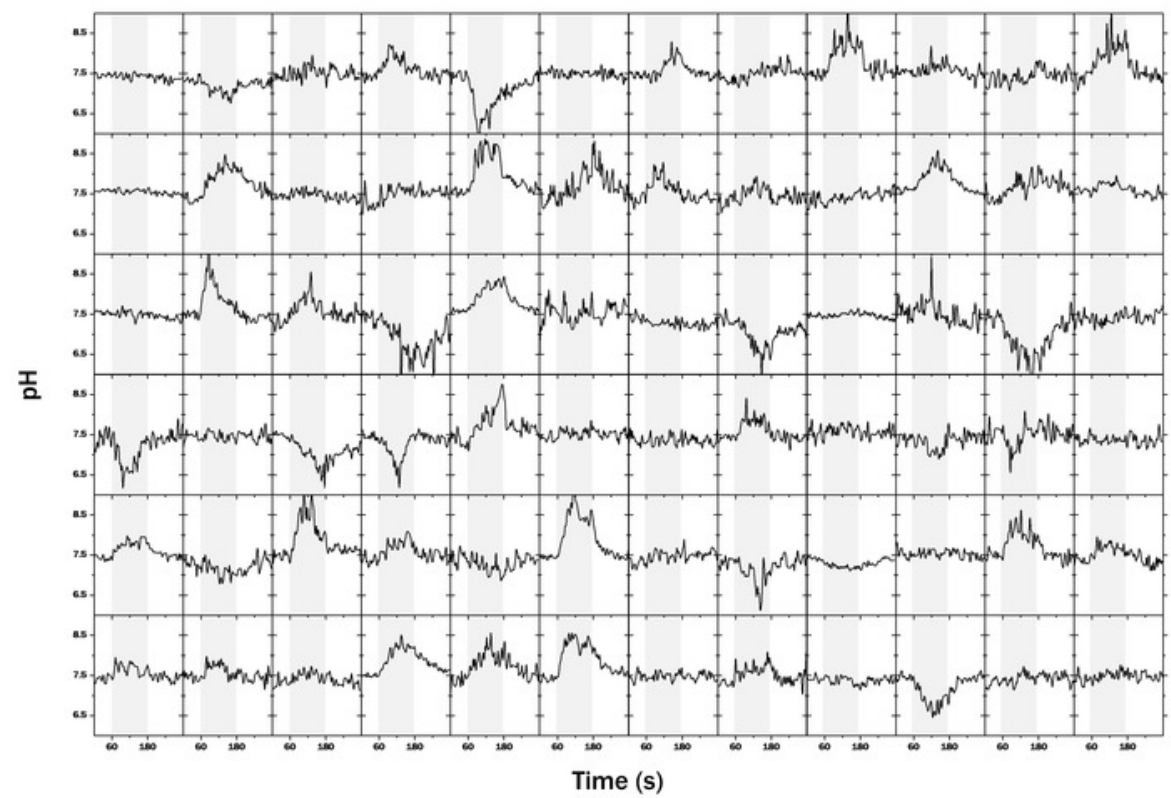

Figure 5: $\mathrm{pH}$ traces of liposomes containing 1.3\% of enzyme. Traces of $\mathrm{pH}$ change of 72 single liposomes, randomly selected, containing cytochrome $\mathrm{bo}_{3}(1.3 \% \mathrm{w} / \mathrm{w})$ measured and analyzed during one experiment. From 0-60 s, no potential is applied (OCP); between 60 and $180 \mathrm{~s}$ (grey zone) -0.2 V vs. SHE; between 180 and 300 s, $0.4 \mathrm{~V}$ vs. SHE was applied. Please click here to view a larger version of this figure. 


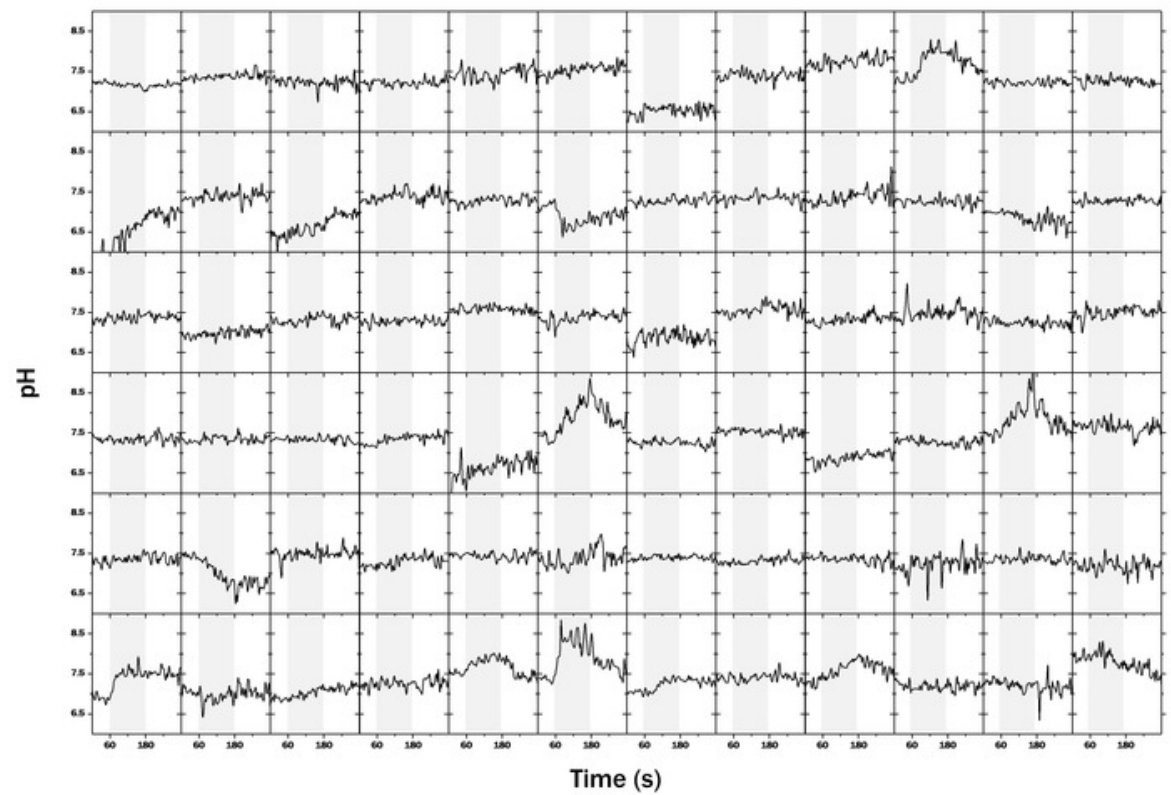

Figure 6: $\mathrm{pH}$ traces of liposomes containing $0.1 \%$ of enzyme. Traces of $\mathrm{pH}$ change of 72 single liposomes, randomly selected, containing cytochrome $\mathrm{bo}_{3}(0.1 \% \mathrm{w} / \mathrm{w})$ measured and analyzed during one experiment. From 0-60 s, no potential is applied (OCP); between 60 and $180 \mathrm{~s}$ (grey zone) $-0.2 \mathrm{~V}$ vs. SHE; between 180 and $300 \mathrm{~s}, 0.4 \mathrm{~V}$ vs. SHE was applied. Please click here to view a larger version of this figure.

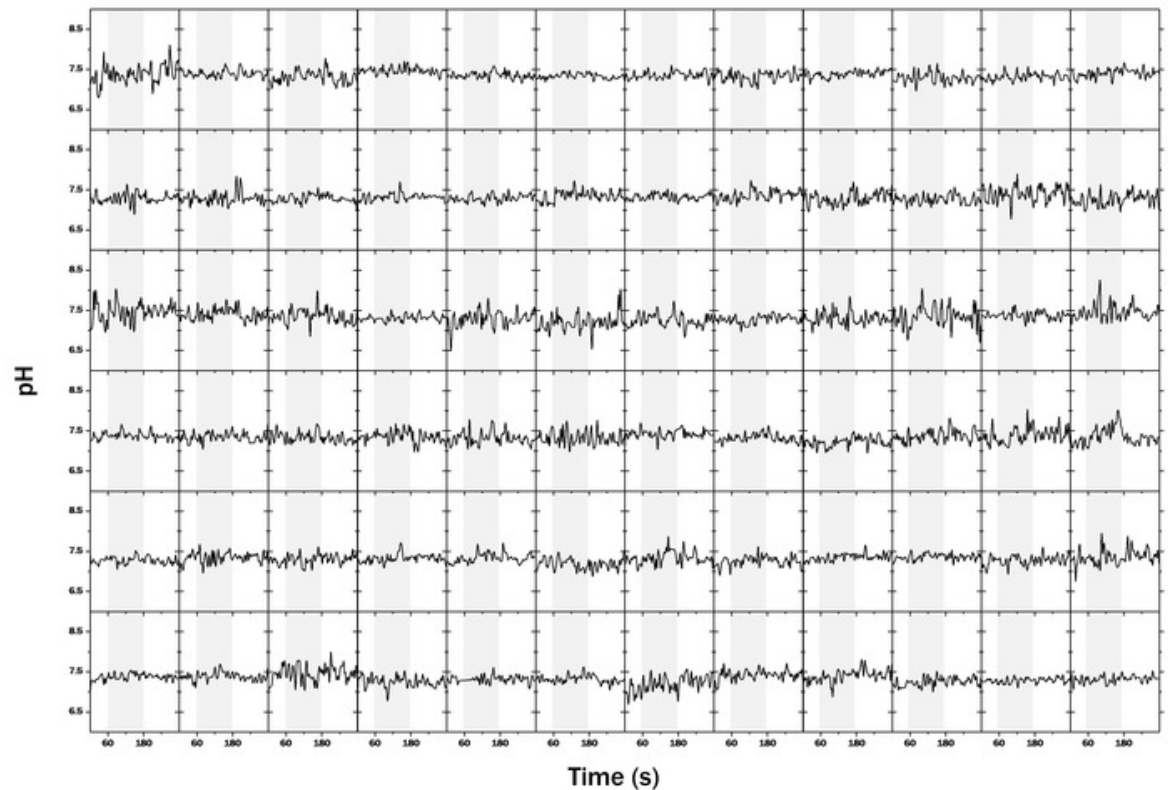

Figure 7: pH traces of liposomes without enzyme. Traces of $\mathrm{pH}$ change of 72 single liposomes, randomly selected, without cytochrome bo 3 measured and analyzed during one experiment. From 0-60 s: no potential is applied (OCP); between 60 and $180 \mathrm{~s}$ (grey zone) $-0.2 \mathrm{~V}$ vs. SHE; between 180 and 300 s, $0.4 \mathrm{~V}$ vs. SHE was applied. Please click here to view a larger version of this figure. 


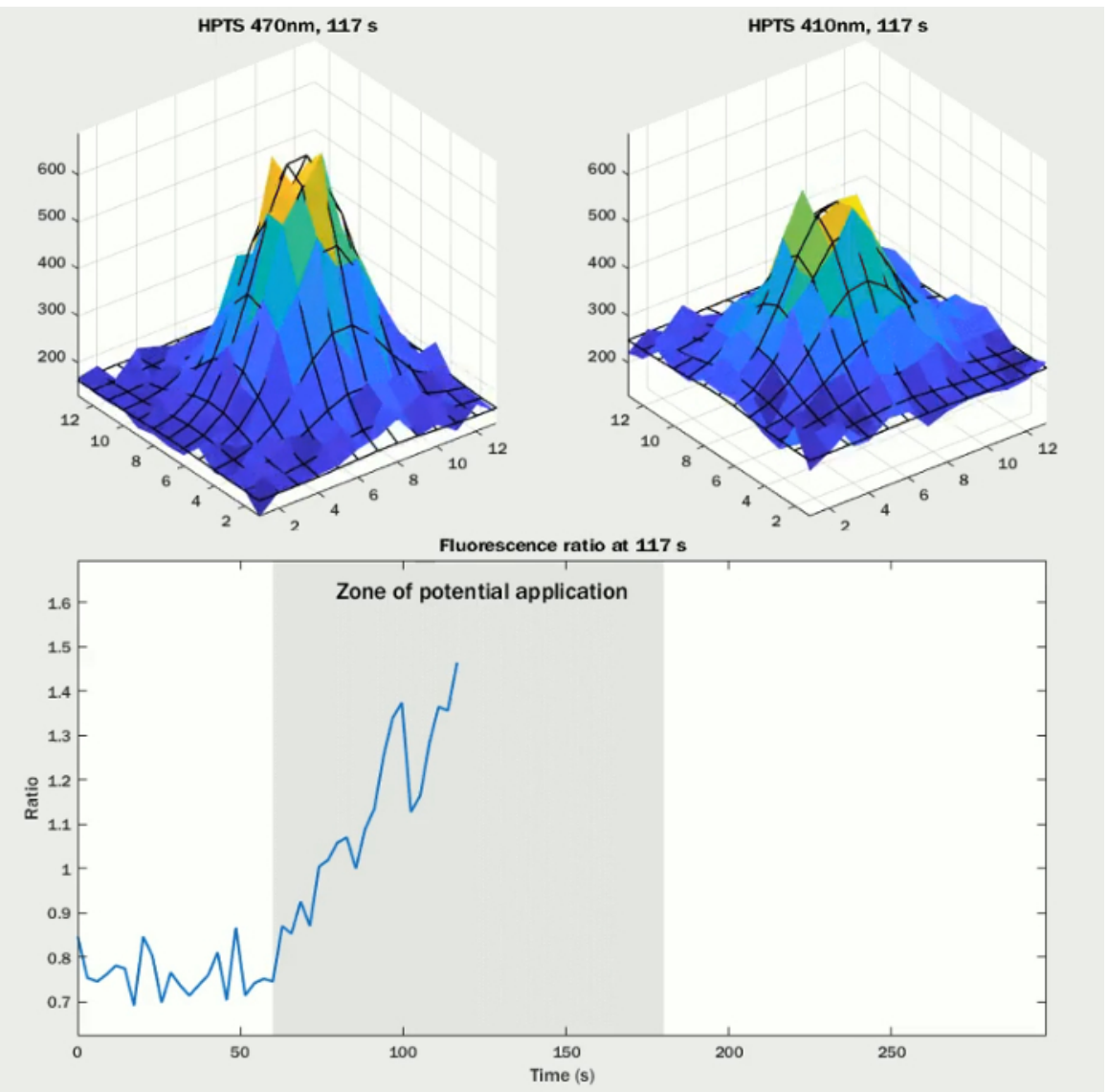

Movie 1: Animation of single liposome pH change. (top panel) Change of a liposome fluorescence on two HPTS channels (shown as 3Dsurface plot of the corresponding area) during $300 \mathrm{~s}$ of the experiment. The reductive potential was applied between $60 \mathrm{~s}$ and $180 \mathrm{~s}$. (bottom panel) Corresponding plot of volumetric intensity ratio of two HPTS channels versus time. The zone of reductive potential application is shaded in grey. Please click here to view this video. (Right-click to download.)

Supplemental Files. Please click here to download the files.

\section{Discussion}

The method described is suitable to study proton pumping by respiratory membrane proteins that can be reconstituted into liposomes and are able to exchange electrons with the quinone pool. Proton pumping activity can be monitored at the single-enzyme level using pH-sensitive (ratiometric) dyes encapsulated in the liposome lumen (Figure 1A).

The method relies on the ability of ubiquinone (or other quinones), incorporated into the lipid bilayer, to exchange electrons with electrodes modified with a SAM ${ }^{18}$. The properties of the gold electrode, modified with a SAM, are very specific. Liposomes need to adsorb onto the SAM and the SAM needs to be thin enough to enable rapid electrochemical oxidation and reduction of the quinone pool in the liposomes. Importantly, the interaction between the electrode and liposome needs to be weak enough not to impair the integrity of the lipid membrane. Furthermore, depending on the details of the experimental system, it is desirable if the SAM prevents gold-catalyzed side reactions, such as oxygen reduction. Finally, the SAM needs to be stable within the potential window used. The use of ultra-flat gold electrodes modified with high-quality SAM of $6 \mathrm{MH}$ fulfills these requirements in the case of cytochrome $b_{3}$. Stripping gold electrodes from atomically-flat silicon wafers creates an ultrasmooth gold surface with a near ideal SAM as indicated by the impedance spectra. Please note that we form the SAM from $6 \mathrm{MH}$ in water. We previously reported on SAMs with $6 \mathrm{MH}$ in isopropanol ${ }^{16}$, but these SAMs exhibit higher double-layer capacitance values and impedance spectra that indicate a more heterogeneous surface (i.e., more defects) with a lower resistance. Furthermore, when SAMs from 6MH are prepared from a water solution, less background oxygen reduction (due to gold catalyzed oxygen reduction) is observed compared to SAMs formed from an ethanol/isopropanol solution. All these observations indicate that SAMs from $6 \mathrm{MH}$ in water solutions have a higher quality with less defects. Higher-quality SAMs can also be formed from thiol-compounds with longer alkane chains (e.g., 1-hydroxy-decane-thiol), but the electron transfer kinetics become slower as the SAM thickness increases.

During (spectro)electrochemistry, chloride ions should be avoided in the buffer solution since they may chemosorb on the gold surface at high potentials, possibly deteriorating the SAM quality. For the same reason, a chloride-free reference electrode should be preferred.

Another important point is the background permeability of the liposomes to protons, which should be low enough to allow proton accumulation as a result of enzymatic proton translocation on the timescale of experiment. The exact lipid composition may influence this permeability. In our work, we use polar $E$. coli lipid extracts supplemented with ubiquinone-10. Proton permeability has been shown to depend strongly on the fatty-acid chain length ${ }^{25}$, although this has been contradicted in studies with more complex lipid compositions ${ }^{26}$. Interestingly, ubiquinone has 
recently been shown to enhance membrane stability ${ }^{27}$. Finally, residual detergents from the protein reconstitution procedure can influence proton leakage and hence it is important to remove detergents as completely as possible using hydrophobic polystyrene microbeads, dilution followed by centrifugation and thorough rinsing of the electrochemical cell after the proteoliposomes are adsorbed on surface. If doubtful, liposome permeability can be verified by following the lumen $\mathrm{pH}$ change (via HPTS) in a response to an external $\mathrm{pH}$ jump ${ }^{28}$.

Single enzyme measurement require unilamelar liposomes and smaller liposomes are expected to show faster or higher $\mathrm{pH}$ changes as the lumen's volume decreases. To achieve this, polystyrene microbeads are added gradually in small amounts leading to slow rate of liposomes formation. In such conditions, small liposomes of homogeneous size are formed with an average diameter of $70 \mathrm{~nm}$ and a polydispersity index of 0.24 in our case ${ }^{14}$. HPTS also adsorbs on the polystyrene microbeads, reducing the capacity of polystyrene microbeads to adsorb detergent. Hence, excess polystyrene microbeads should be added to compensate for its loss. Treatment with polystyrene microbeads was observed to reduce the HPTS concentration in the lipid-protein suspension by about a quarter (from $5 \mathrm{mM}$ to $3-4 \mathrm{mM}$ ). However, the actual HPTS concentration in the liposome's lumen might be higher since the liposomes are formed early on the treatment with polystyrene microbeads. Instead of HPTS, other $\mathrm{pH}$-sensitive dyes can be used. However, it is important that the dye is membrane-impermeable and ratiometric. The latter avoids experimental errors due to photobleaching and varying amounts of encapsulated dye.

Simple calculations can be made to estimate whether, stochastically, most of the non-empty liposomes contain only one enzyme molecule. Assuming an average liposome diameter of $70 \mathrm{~nm}$, a lipid molecular weight of $750 \mathrm{Da}$ and a lipid area of $0.65 \mathrm{~nm}^{2}$ gives us a liposome mass of $5.2 \times 10^{-17} \mathrm{~g}$, i.e., $9.6 \times 10^{13}$ liposomes per preparation ( $5 \mathrm{mg}$ of lipid). At $0.1 \%$ of cytochrome $b o_{3}(\mathrm{MW}=144 \mathrm{kDa}), 2 \times 10^{13}$ enzyme molecules are present, corresponding to a 0.2 protein:liposome ratio. Using a Poisson distribution, one may further calculate that the probability to find one enzyme molecule (17\%) per liposome is ten times higher than probability to find more than one molecule $(1.7 \%)$. More precise calculations can be made if the losses of enzyme and lipids during the reconstitution determined from corresponding assays are taken into account. The latter can be estimated from a protein assay and by measuring FDLL fluorescence of prepared liposomes.

Once the protocol is established and single enzyme traces are recorded, further modifications of the method is feasible depending on the aim. One might think about an enzyme inhibitor addition or introduction of an ionophore into the system. Care should be taken to verify that addition of solvents used to prepare ionophore or inhibitor stock do not influence the state of SAM or permeability of the liposomes.

The technique as described here is limited to a particular group of enzymes that are both membrane proton transporters and quinone-converting. The equipment and experimental conditions as used here were optimized for the enzymatic activity of cytochrome bo ${ }_{3}$. Here, the time resolution is 2-3 seconds, determined by the exposure time and the time it takes for the turret to change filters. The duration of the experiment is limited by photobleaching of fluorescent dye and, thus, by the light intensity. We have previously found average proton translocation rate of cytochrome $\mathrm{bO}_{3}$ to be $73 \pm 2.2$ protons/s using this technique, although activities down to 20 protons/s were detected. To use these techniques for enzymes with either more or less activity, image acquisition parameters need to be adapted (i.e., light intensity, exposure time and duration of experiment). In the future, this method can be extended towards other electron transport driving proton pumping enzymes, an example being mitochondrial complex I. Other enzymes might require different reconstitution protocols, and this would need to be optimized for each different transporter. Proton pumps that are not quinone-converting enzymes can also be studied, e.g., ATP-driven ${ }^{29}$, although in this case proton translocation cannot be triggered electrochemically, but addition of an initiator (e.g., ATP) is required. In the latter case, there is no need to use a gold-modified cover slip. Moreover, provided that a suitable membrane-impermeable and ion-sensitive fluorescent dye is used, this method can be extended to other ionic pumps, e.g., sodium and potassium.

\section{Disclosures}

The authors have nothing to disclose.

\section{Acknowledgments}

The authors acknowledge the BBSRC (BB/P005454/1) for financial support. NH was funded by the VILLUM Foundation Young Investigator Program.

\section{References}

1. Claessen, V.I. et al. Single-Biomolecule Kinetics: The Art of Studying a Single Enzyme. Annual Review of Analytical Chemistry. 3 (1), $319-340$ (2010).

2. Rojek, M.J., Walt, D.R. Observing Single Enzyme Molecules Interconvert between Activity States upon Heating. PLoS ONE. 9 (1), e86224 (2014).

3. Velonia, K. et al. Single-Enzyme Kinetics of CALB-Catalyzed Hydrolysis. Angewandte Chemie International Edition. 44 (4), $560-564$ (2005).

4. Lu, H.P., Xun, L., Xie, X.S. Single-molecule enzymatic dynamics. Science (New York, N.Y.). 282 (5395), 1877-82 (1998).

5. Engelkamp, H., Hatzakis, N.S., Hofkens, J., De Schryver, F.C., Nolte, R.J.M., Rowan, A.E. Do enzymes sleep and work? Chemical Communications. (9), 935-940 (2006).

6. Boukobza, E., Sonnenfeld, A., Haran, G. Immobilization in Surface-Tethered Lipid Vesicles as a New Tool for Single Biomolecule Spectroscopy. The Journal of Physical Chemistry B. 105 (48), 12165-12170 (2001).

7. Hsin, T.-M., Yeung, E.S. Single-Molecule Reactions in Liposomes. Angewandte Chemie International Edition. 46 (42), $8032-8035$ (2007).

8. García-Sáez, A.J., Schwille, P. Single molecule techniques for the study of membrane proteins. Applied Microbiology and Biotechnology. 76 (2), 257-266 (2007).

9. Onoue, Y. et al. A giant liposome for single-molecule observation of conformational changes in membrane proteins. Biochimica et Biophysica Acta (BBA) - Biomembranes. 1788 (6), 1332-1340 (2009).

10. Jefferson, R.E., Min, D., Corin, K., Wang, J.Y., Bowie, J.U. Applications of Single-Molecule Methods to Membrane Protein Folding Studies. Journal of Molecular Biology. 430 (4), 424-437 (2018). 
11. Rigaud, J.L., Pitard, B., Levy, D. Reconstitution of membrane proteins into liposomes: application to energy-transducing membrane proteins. BBA - Bioenergetics. 1231 (3), 223-246 (1995).

12. Jesorka, A., Orwar, O. Liposomes: Technologies and Analytical Applications. Annual Review of Analytical Chemistry. 1 (1), 801-832 (2008).

13. Seddon, A.M., Curnow, P., Booth, P.J. Membrane proteins, lipids and detergents: Not just a soap opera. Biochimica et Biophysica Acta Biomembranes. 1666 (1-2), 105-117 (2004).

14. Li, M. et al. Single Enzyme Experiments Reveal a Long-Lifetime Proton Leak State in a Heme-Copper Oxidase. Journal of the American Chemical Society. 137 (51), 16055-16063 (2015).

15. Rumbley, J.N., Furlong Nickels, E., Gennis, R.B. One-step purification of histidine-tagged cytochrome bo3 from Escherichia coli and demonstration that associated quinone is not required for the structural integrity of the oxidase. Biochimica et Biophysica Acta (BBA) - Protein Structure and Molecular Enzymology. 1340 (1), 131-142 (1997).

16. Jeuken, L.J.C. et al. Phase separation in mixed self-assembled monolayers and its effect on biomimetic membranes. Sensors and Actuators, B: Chemical. 124 (2), 501-509 (2007).

17. Barsoukov, E., Macdonald, J.R. Impedance Spectroscopy Theory, Experiment, and Applications. Chapters 1-2. John Wiley \& Sons. (2005).

18. Jeuken, L.J.C., Connell, S.D., Henderson, P.J.F., Gennis, R.B., Evans, S.D., Bushby, R.J. Redox Enzymes in Tethered Membranes. Journal of the American Chemical Society. 128 (5), 1711-1716 (2006).

19. Compton, R.G., Banks, C.E. Understanding voltammetry. Chapter 4. World Scientific Publishing Europe Ltd. Singapore. (2018).

20. Girault, H.H. Analytical and Physical Electrochemistry. Chapter 10. EPFL Press. Lausanne. (2004).

21. Mazurenko, I., Jeuken, L.J.C. Timelapse (movie) of single vesicles fluorescence change upon potential application. (2018).

22. Li, M., Khan, S., Rong, H., Tuma, R., Hatzakis, N.S., Jeuken, L.J.C. Effects of membrane curvature and pH on proton pumping activity of single cytochrome bo3enzymes. Biochimica et Biophysica Acta - Bioenergetics. 1858 (9), 763-770 (2017).

23. Li, M., Tuma, R., Jeuken, L.J.C. MATLAB code for the analysis of proton transport activity in single liposomes. (2017).

24. Li, M., Tuma, R., Jeuken, L.J.C. Time-resolved fluorescence microscopic data (traces) of individual lipid vesicles with proton transport activity. (2017).

25. Paula, S., Volkov, A.G., Van Hoek, A.N., Haines, T.H., Deamer, D.W. Permeation of protons, potassium ions, and small polar molecules through phospholipid bilayers as a function of membrane thickness. Biophysical Journal. 70 (1), 339-348 (1996).

26. Brookes, P.S., Hulbert, A.J., Brand, M.D. The proton permeability of liposomes made from mitochondria) inner membrane phospholipids: No effect of fatty acid composition. Biochimica et Biophysica Acta (BBA) - Biomembranes. 1330 (2), 157-164 (1997).

27. Eriksson, E.K., Agmo Hernández, V., Edwards, K. Effect of ubiquinone-10 on the stability of biomimetic membranes of relevance for the inner mitochondrial membrane. Biochimica et Biophysica Acta - Biomembranes. 1860 (5), 1205-1215 (2018)

28. Seneviratne, R. et al. A reconstitution method for integral membrane proteins in hybrid lipid-polymer vesicles for enhanced functional durability. Methods. 1-8 (2018).

29. Veshaguri, S. et al. Direct observation of proton pumping by a eukaryote P-type ATPase. Science. 351 (6280), 1-6 (2016). 